\title{
Mode locked fiber laser training kit
}

\section{Sophie Leprevost, Patrice Le Boudec}

Sophie Leprevost, Patrice Le Boudec, "Mode locked fiber laser training kit," Proc. SPIE 9664, Ninth International Topical Meeting on Education and Training in Optics and Photonics, 96642H (24 October 2005); doi: $10.1117 / 12.2207570$

SDIE Event: Ninth International Topical Meeting on Education and Training in Optics and Photonics, 2005, Marseille, France 
Ref ETOP019

\title{
Mode locked fiber laser training kit
}

\author{
Sophie LEPREVOST \& Patrice LE BOUDEC
}

\begin{abstract}
This paper presents kits dedicated to optical phenomena education proposed by Idil Fibres Optiques. It shows the teaching means used to guarantee students the best understanding of physical phenomena related to optical fibers. In a second part we present one of these kits : the mode-locked fiber laser. This kit allows in particular to understand the effects of chromatic dispersion, non-linearity and mode-locking during the ultra-short pulse generation in mode-locked fiber lasers with "figure-8" and unidirectional ring cavities.
\end{abstract}

\section{Summary}

\section{Presentation of the educational and training kits}

Idil Fibres Optiques is specialized in optical fibers engineering and lasers. In particular, it develops educational kits completely adapted to the teaching of the physical phenomena related to optical fibers for engineers and technicians. The range of Idil products are related to the teaching of physical phenomena using fiber optic devices. Our kits encompass WDM, reflectometry, interferometry, telecommunications, EDFAs and EDFLs and, of course, mode-locked fiber lasers.

In our kits, all the optical functions necessary for handling are separated, and the students connect them together via optical patchcords. Our kits use E2000 optical connectors assembled on fiber ends (which guarantee an optimal ocular safety because they close automatically upon extraction). The interest for the students, is to be able to characterize each component separately, to understand its function before combining it with other components and creating a new optical function. Finally, the entire range of educational kits include complete documentation presenting the theory of the physical phenomena approached in the training. All of our documentation (theory and practical) is written in collaboration with optics scientists and professors in order to guarantee a clear understanding of the subject matter.

\section{Presentation of the mode locked fiber laser kit}

One of the latest kits developed by Idil involves the study of an ultra-short pulse fiber laser generated by passive mode-locking. This type of laser is currently the subject of research in many laboratories. Mode-locked lasers are used in a wide range of applications, depending on the wavelength and pulse width. They can be used as sources in communications systems for time-division multiplexing (TDM) or wavelengthdivision multiplexing (WDM), as spectroscopic tools in the laboratory for time-resolved studies of fast nonlinear phenomena in semiconductors, as a source for pulsed sensors, or as tunable seed pulses for lasers in medical applications. Applications such as optical coherence tomography could take advantage of the broad bandwidth of a mode-locked fiber laser rather than the temporal ultra-short pulse width. 
The aim of this kit is to observe and study the generation of ultra-short pulses, and in particular the effects of polarization, dispersion, non-linearity and mode-locking. It is straightforward to assemble a "Figure-8" laser or a unidirectional fiber ring laser. Once assembled, the lessons are to observe the locking of the laser, to measure the repetition rate of the laser, to observe the operating modes of the laser : continuous and pulsed (stretched-pulses, solitons, parabolic), to study the effect of the fiber chromatic dispersion behavior of the laser and to observe the various optical spectra.

The unidirectional fiber ring laser (also called polarization additive pulse mode-locking, P-APM or Kerr mode-locking) which is a laser with passive mode-locking, uses the nonlinear polarization rotation. This rotation relies on the Kerr effect in optical fibers in conjunction with polarizer. This causes artificial saturable absorber action and achieves pulse shortening.

Mode-locked lasers in a unidirectional ring configuration are selfstarting if the polarization is well directed. The addition of various lengths of SMF-28 fiber with positive chromatic dispersion will change the sign and the value of the total chromatic dispersion of the cavity. Various types of pulses can be observed : stretchedpulses, solitons, parabolic pulses. A study of pulses duration, energy, width and shape of their spectrum and finally the repetition rate of the pulses (which is proportional to a multiple of the cavity length). The total chromatic dispersion of the ring cavity is

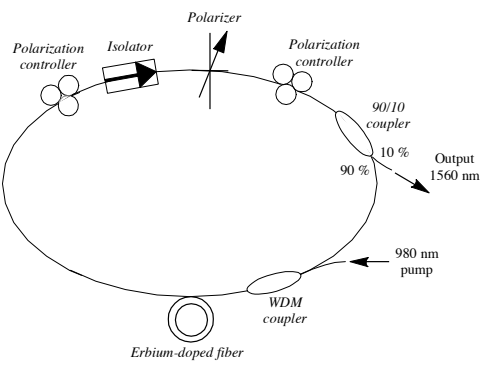

Fig. 1 : All-fiber ring laser mode-lodked by P-A PM negative (normal dispersion) to $1550 \mathrm{~nm}$. It is possible to add an adjusted length of single mode fiber with positive dispersion to obtain a zero chromatic dispersion of the cavity. $300 \mathrm{~m}$ long SMF-28 fiber can also be added to get a positive chromatic dispersion (anomalous dispersion).

The additional fiber in the cavity will modify the total chromatic dispersion, but also modifies the repetition rate of the ultra-short pulses which is proportional to a multiple of the cavity length.

When the total cavity dispersion is positive (anomalous), the laser is in a soliton regime. In this type of mode, the laser tends to create multiple pulses when the pump power increases. For high pump power, the peak power of the soliton will eventually reach the limit imposed by APM saturation. Then the single pulse per cavity round-trip will break up into multiple, uncontrolled pulses. This phenomenon is observed in the "figure-8" lasers and in the long ring lasers. However, the fiber soliton laser can operate with a stable and single pulse per round trip if the cavity is as short as possible (but always with anomalous dispersion) and that the

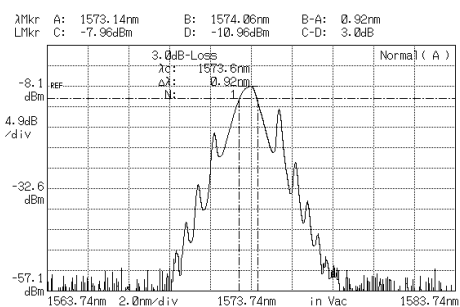

Fig. 2 : Snectrum from a soliton gain is low enough.

The mode-locked pulse spectrum of an all-fiber laser shows significant spectral structure related to the soliton-like nature of the pulse : the Kelly sidebands (cf figure 2). These sidebands are resonant instabilities that can be explained by simple phase-matching. 
When a soliton propagates in a fiber laser, it encounters various periodic perturbations such as gain, filtering and losses due to splices, connectors and intrinsic losses of the components. The perturbed soliton sheds dispersive radiations as it is reshaped back into a proper soliton. Each frequency component of these radiations propagates at its own phase velocity and generates radiations that interfere in a destructively except at frequencies that are phase-matched. This causes sidebands around the centre of the spectrum : the Kelly sidebands. The shorter the pulse width, the nearest from the center the sidebands will be found. Note that the amount of continuum that is generated at a certain frequency is proportional to the spectral amplitude of the soliton at this frequency. The sidebands appear as a pedestal under the pulse and can contain an energy comparable to the pulse energy. The effect of the sidebands can be avoided by decreasing the average dispersion of the cavity; it also allows to decrease the pulse width but it will limit the peak power of the pulse.

In the stretched-pulse laser (cf. figure 3), the lengths of large positive and negative dispersion fiber cause the pulse width to be alternately stretched and compressed as they circulate in the cavity. And thus the average maximum power in the laser is lowered significantly. The net nonlinear phase shift per pass is lower and APM saturation is avoided. Spectral broadening occurs in the doped fiber due to the action of the negative dispersion with self-phase modulation. At the output, the pulses can then have high energy and a broad bandwidth. The stretched-pulse technique yields shorter and more intense pulses than the pulses in a soliton laser and the spectra (cf. figure 4) are broader and cleaner than for soliton.

\section{References}

1. Compact Sources of Ultrashort Pulses, Cambridge Studies in Modern Optics edited by IRL N. Duling, III and Michael L. Dennis

2. Ultrashort-pulse fiber ring lasers, L.E. Nelson, D.J. Jones, K. Tamura, H.A. Haus, E.P. Ippen, Appl. Phys. B 65, 277294 (1997)

3. Self-Similar Evolution of Parabolic Pulses in a Laser, F. Ö. Ilday, J. R. Buckley, and F. W. Wise, Phys. Rev. Lett. 92, 213902 (2004)

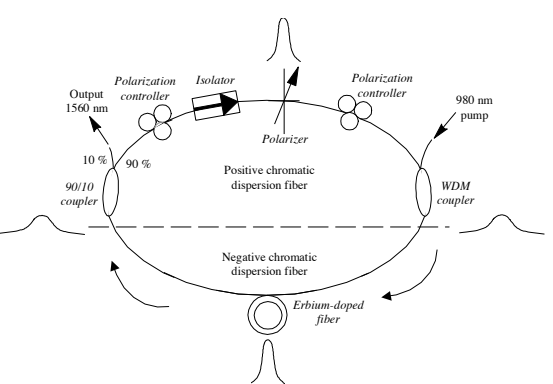

Fig. 3 : All-fiber stretched pulse laser

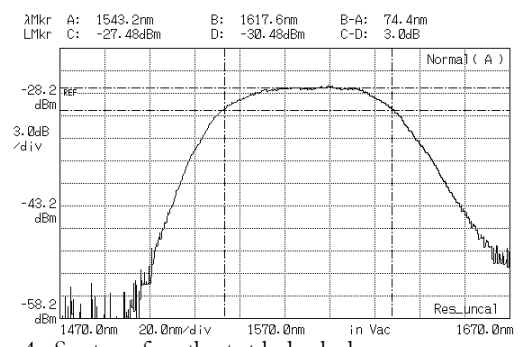

Fig. 4 : Spectrum from the stretched-pulse laser

Anndxidth. $75 \mathrm{~nm}$ cimifimntly hroder than in fimure- 8 lacer 